\title{
Mehran in-stent restenosis classification adapted for coronary bifurcations: the impact on 4-year follow-up from randomized clinical studies POLBOS I and II
}

\author{
Jacek Bil ${ }^{1}$, Robert J. Gil ${ }^{1}$, Adam Kern², Luis A. Inigo-Garcia ${ }^{3}$, Radoslaw Formuszewicz ${ }^{4}$, Slawomir Dobrzycki ${ }^{5}$ \\ ${ }^{1}$ Department of Invasive Cardiology, Central Clinical Hospital of the Ministry of Interior and Administration, Warsaw, Poland \\ ${ }^{2}$ Department of Cardiology and Cardiosurgery, University of Warmia and Mazury, Olsztyn, Poland \\ ${ }^{3}$ Department of Cardiology, Costa del Sol Hospital, Marbella, Spain \\ ${ }^{4}$ Department of Cardiology, $10^{\text {th }}$ Clinical Military Hospital, Bydgoszcz, Poland \\ ${ }^{5}$ Department of Invasive Cardiology, Medical University of Bialystok, Bialystok, Poland
}

Adv Interv Cardiol 2018; 14, 3 (53): 299-303

DOI: https://doi.org/10.5114/aic.2018.78335

\section{Introduction}

Percutaneous coronary interventions $(\mathrm{PCl})$ with stent deployment are the most widely performed procedures in the therapy of symptomatic coronary artery disease (CAD). In the last three decades, PCI with stent deployment has changed the practice in cardiology. Drug-eluting stents (DES) significantly reduced in-stent restenosis (ISR) rates - one of the key limitations of bare metal stents. In consequence, DES were rapidly and widely accepted, which allowed more complex coronary interventions, including bifurcations, to be performed. Nevertheless, ISR has remained a troublesome late stent complication [1, 2].

Multiple classification systems addressed the problem of ISR severity. The Mehran classification is a morphologic system which divides ISR lesions into four patterns: from focal pattern I when ISR is $\leq 10 \mathrm{~mm}$ in length within the stent to pattern IV when the ISR is the cause of vessel occlusion [3].

\section{Aim}

The aim of our study was to propose a modified Mehran restenosis classification adapted to bifurcation lesions and preliminarily assess its value in the 4-year follow-up on data from two randomized studies, POLBOS I and POLBOS II, that compared dedicated bifurcation BiOSS stents with regular drug-eluting stents (rDES) [4-6].

\section{Material and methods \\ POLBOS I and POLBOS II were international, multi- center, randomized, open-label, controlled studies de-}

scribed previously $[4,6]$. Briefly, the inclusion criteria were: stable CAD or non-ST-segment elevation acute coronary syndrome (NSTE-ACS), age $\geq 18$ years, de novo coronary bifurcation lesion, main vessel (MV) diameter $\geq 2.5 \mathrm{~mm}$, and side branch (SB) diameter $\geq 2.0 \mathrm{~mm}$ on visual estimation. The Institutional Review Board of each participating center approved the study protocol (ClinicalTrials.gov Identifier: POLBOS I - NCT02192840, POLBOS II - NCT02198300).

After providing written informed consent, patients were randomly assigned to one of two treatment strategies: BiOSS Expert (in POLBOS I)/BiOSS LIM (in POLBOS II) stent implantation or rDES implantation [7-9]. Provisional T-stenting was the default strategy. The stent nominal diameter was chosen according to the distal reference, and after stent deployment, the proximal part of the stent was optimized, if needed, with proximal optimization technique (POT) to obtain the proper apposition.

Clinical follow-up was performed by telephone 1 , $6,12,24,36$ and 48 months after the procedure. Adverse events were monitored throughout the study period. Follow-up coronary angiography was mandatory at 12 months unless clinically indicated earlier.

The primary endpoint was the cumulative rate of major adverse cardiovascular events (MACE) consisting of cardiac death, myocardial infarction (MI), and target lesion revascularization (TLR). Secondary endpoints included cardiac death, all-cause death, MI, TLR, target vessel revascularization (TVR), stent thrombosis (ST), and device success. Cardiac death included death resulting from an acute MI, sudden cardiac death, death due to

\section{Corresponding author:}

Jacek Bil MD, PhD, Department of Invasive Cardiology, Central Clinical Hospital of the Ministry of Interior and Administration, 137 Woloska St, 02-507 Warsaw, Poland, phone: +48 2250816 86, e-mail: biljacek@gmail.com

Received: 11.02.2018, accepted: 9.04.2018. 
heart failure, and death due to cardiac procedures. All deaths were deemed cardiac unless proven otherwise. Myocardial infarction was defined according to the third universal definition [10].

Bearing in mind the different approach for bifurcation stenting (nominal stent diameter chosen on the basis of distal reference diameter and then optimization of the proximal part of the stent with POT and final kissing bal- lons technique (FKB)) we proposed a modified Mehran restenosis classification adapted for coronary bifurcation lesions (Figure 1). We divided the stent according to the parts of the bifurcation, i.e. the MV and main branch $(\mathrm{MB})$, and additionally we took into consideration the SB since it is an inseparable part of the bifurcation complex. In each pattern (I-IV) we introduced subgroups to localize the restenosis (in $\mathrm{MV}$, in $\mathrm{MB}$, in $\mathrm{SB}$, or in combina-

Modified Mehran's classification adapted for restenosis in bifurcation lesions

\begin{tabular}{|c|c|c|}
\hline Type of restenosis & BiOSS $(n=24)$ & rDES $(n=17)$ \\
\hline I (focal restenosis, < $10 \mathrm{~mm}$ in stent): & $14(58.3 \%)$ & $9(52.9 \%)$ \\
\hline $\mathrm{A}($ in $M V)$ & $4(28.6 \%)$ & $2(11.8 \%)$ \\
\hline$B($ in $M B)$ & $2(14.3 \%)$ & $1(5.9 \%)$ \\
\hline$C($ in $S B)$ & $5(20.8 \%)$ & $3(17.7 \%)$ \\
\hline $\mathrm{D}$ (in $\mathrm{SB}$ and $\mathrm{MB}$ ) & $1(4.2 \%)$ & $2(11.8 \%)$ \\
\hline $\mathrm{E}$ (in SB and MV or in all parts) & $2(14.3 \%)$ & $1(5.9 \%)$ \\
\hline II (> $10 \mathrm{~mm}$ within the stent): & $5(20.8 \%)$ & $5(29.4 \%)$ \\
\hline $\mathrm{A}($ in $\mathrm{MV}$ ) & $1(4.2 \%)$ & $1(5.9 \%)$ \\
\hline$B($ in $M B)$ & $1(4.2 \%)$ & 0 \\
\hline$C($ in SB) & 0 & 0 \\
\hline $\mathrm{D}$ (in SB and MB) & $2(14.3 \%)$ & $2(11.8 \%)$ \\
\hline $\mathrm{E}$ (in SB and MV or in all parts) & $1(4.2 \%)$ & $2(11.8 \%)$ \\
\hline III (> $10 \mathrm{~mm}$ + outside the stent): & $2(8.3 \%)$ & $2(11.8 \%)$ \\
\hline $\mathrm{A}($ in $M V)$ & $1(4.2 \%)$ & $1(5.9 \%)$ \\
\hline$B($ in $M B)$ & 0 & 0 \\
\hline$C$ (in SB) & 0 & 0 \\
\hline $\mathrm{D}$ (in SB and MB) & $1(4.2 \%)$ & $1(5.9 \%)$ \\
\hline $\mathrm{E}$ (in SB and MV or in all parts) & 0 & 0 \\
\hline IV (total occlusion): & $3(12.5 \%)$ & $1(5.9 \%)$ \\
\hline A (in MV) & 0 & 0 \\
\hline$B($ in $M B)$ & $1(4.2 \%)$ & $1(5.9 \%)$ \\
\hline$C($ in SB) & $1(4.2 \%)$ & 0 \\
\hline D (in MB and SB) & $1(4.2 \%)$ & 0 \\
\hline \multicolumn{3}{|l|}{ Exemplary cases: } \\
\hline & BiOSS: ISR type III [ & rDES: ISR type I \\
\hline
\end{tabular}

Figure 1. Modified Mehran restenosis classification. In each pattern (I-IV) we introduced subgroups to localize the restenosis (in MV-MB, in SB, or in both, respectively). Point IIC is optional depending on the SB stenting, in other cases SB restenosis characterizes the lesion irrespectively of whether it was stented or not since SB is an inseparable part of the bifurcation complex

$M V$ - main vessel, $M B$ - main branch, SB - side branch. 
tions). The MV was defined as the proximal part of the bifurcation up to the take-off of the SB, and the MB was defined as the distal part of the bifurcation below the take-off of the SB.

\section{Statistical analysis}

Continuous variables were presented as mean \pm standard deviation. Categorical data were presented as numbers (\%). Continuous variables were compared using an unpaired two-sided Student $t$-test, and categorical data using the $\chi^{2}$ test or Fisher's exact test, as appropriate. If the distribution was not normal on the Shapiro-Wilk test, the Wilcoxon signed-rank and Mann-Whitney U-tests were used. $P$-values of $<0.05$ were considered statisti- cally significant. The significance level was set at 0.05. Statistical analyses were performed using R 3.0.2 for OS (R Foundation, Vienna, Austria).

\section{Results}

Our population of 445 patients, with 222 patients in the BiOSS group and 223 patients in the rDES group, was analyzed. In the BiOSS group there were $24(10.8 \%)$ cases of restenosis and in the rDES group 17 (7.6\%) cases at 12-month follow-up (the rate of angiographic control was $90.3 \%$ ). Baseline clinical and procedural characteristics of patients with restenosis are presented in Table I. In the rDES group in patients with restenosis there was a higher rate of diabetes type 2 (33\% vs. $52.9 \%, p<0.05)$

Table I. Baseline population characteristics in the whole population

\begin{tabular}{|c|c|c|c|c|}
\hline \multirow[t]{2}{*}{ Parameter } & \multicolumn{2}{|c|}{ BiOSS group } & \multicolumn{2}{|c|}{ rDES group } \\
\hline & $\begin{array}{l}\text { No restenosis } \\
\quad(n=198)\end{array}$ & $\begin{array}{c}\text { Restenosis } \\
(n=24)\end{array}$ & $\begin{array}{l}\text { No restenosis } \\
\quad(n=206)\end{array}$ & $\begin{array}{l}\text { Restenosis } \\
(n=17)\end{array}$ \\
\hline \multicolumn{5}{|l|}{ Baseline clinical characteristics: } \\
\hline Age [years] & $66.6 \pm 9.7$ & $65.2 \pm 12.6$ & $66.5 \pm 9.1$ & $65.6 \pm 9.5$ \\
\hline Women & $57(28.8 \%)$ & $5(20.8 \%)$ & $61(29.6 \%)$ & $3(17.6 \%)$ \\
\hline Hypertension & $158(79.8 \%)$ & $22(91.7 \%)$ & $158(76.7 \%)$ & $13(76.5 \%)^{\star \star}$ \\
\hline Hypercholesterolemia & $143(72.2 \%)$ & $17(70.8 \%)$ & $136(66 \%)$ & $14(82.4 \%)^{*}$ \\
\hline Diabetes type 2 & $82(41.4 \%)$ & $8(33.3 \%)$ & $63(30.6 \%)$ & $9(52.9 \%)^{\star, \star \star}$ \\
\hline Prior myocardial infarction & $85(42.9 \%)$ & $14(58.3 \%)^{*}$ & $82(39.8 \%)$ & $8(47.1 \%)$ \\
\hline Prior $\mathrm{PCl}$ & $101(51 \%)$ & $11(45.8 \%)$ & $109(52.9 \%)$ & $6(35.3 \%)^{*}$ \\
\hline Coronary artery bypass graft & $17(8.6 \%)$ & $4(16.7 \%)$ & $19(9.2 \%)$ & $3(17.6 \%)$ \\
\hline Chronic kidney disease & $22(11.1 \%)$ & $1(4.2 \%)$ & $16(7.8 \%)$ & $3(17.6 \%)$ \\
\hline History of smoking & $44(22.2 \%)$ & $3(12.5 \%)$ & $50(24.3 \%)$ & $7(41.2 \%)^{*, \star \star}$ \\
\hline \multicolumn{5}{|l|}{ Clinical indication for $\mathrm{PCl}$ : } \\
\hline Planned PCl & $167(84.3 \%)$ & $19(79.2 \%)$ & $176(85.4 \%)$ & $10(58.8 \%)^{\star, \star \star}$ \\
\hline UA/NSTEMI & $31(15.7 \%)$ & $5(20.8 \%)$ & $30(14.6 \%)$ & $7(41.2 \%)^{*, \star \star}$ \\
\hline True bifurcation & $167(84.3 \%)$ & $11(45.8 \%)^{*}$ & $176(85.4 \%)$ & $9(52.9 \%)^{*}$ \\
\hline Left main bifurcation & $57(28.8 \%)$ & $5(20.8 \%)$ & $51(24.8 \%)$ & $6(35.3 \%)$ \\
\hline \multicolumn{5}{|l|}{ Procedural characteristics: } \\
\hline Main vessel predilatation & $117(59.1 \%)$ & $20(83.3 \%)^{*}$ & $145(70.4 \%)$ & $14(82.4 \%)$ \\
\hline Side branch predilatation & $67(33.8 \%)$ & $7(29.2 \%)$ & $57(27.7 \%)$ & $8(47.1 \%)^{*, \star \star}$ \\
\hline Olimus-eluting stents & $88(44.4 \%)$ & $14(58.3 \%)$ & $148(71.8 \%)$ & $7(41.2 \%)^{*, * \star}$ \\
\hline Paclitaxel-eluting stents & $110(55.6 \%)$ & $10(41.7 \%)$ & $58(28.2 \%)$ & $10(58.8 \%)^{*, \star \star}$ \\
\hline Proximal optimization technique & $81(40.9 \%)$ & $2(8.3 \%)^{*}$ & $152(73.8 \%)$ & $1(5.9 \%)^{\star}$ \\
\hline Final kissing balloon & $65(32.8 \%)$ & $6(25 \%)$ & $101(49 \%)$ & $9(52.9 \%)^{* *}$ \\
\hline Additional stent in side branch & $17(8.6 \%)$ & $5(20.8 \%)$ & $7(3.4 \%)$ & $8(47.1 \%)^{*, * \star}$ \\
\hline
\end{tabular}

PCI - percutaneous coronary intervention, UA/NSTEMI - unstable angina/non-ST-elevation myocardial infarction; ${ }^{*} p<0.05$ no restenosis vs restenosis in BiOSS or rDES group; ${ }^{* *} p<0.05$ restenosis between BiOSS and rDES groups. 
and a history of smoking ( $12.5 \%$ vs. $41.2 \%, p<0.05)$ and a lower rate of hypertension $(91.7 \%$ vs. $76.5 \%, p<0.05)$ compared with the BiOSS group. In the rDES group in patients with restenosis there was a higher rate of SB predilatation ( $29.2 \%$ vs. $47.2 \%, p<0.05)$, final kissing balloon technique ( $25 \%$ vs. $52.9 \%, p<0.05)$, and additional stent in the SB $(20.8 \%$ vs. $47.1 \%, p<0.05)$ compared with the BiOSS group.

The rates of restenosis in the BiOSS group in the $\mathrm{MV}, \mathrm{MB}$ and SB were $41.7 \%(n=10), 37.5 \%(n=9)$ and $54.2 \%(n=13)$, respectively, whereas rates of restenosis in the rDES group in the MV, MB and SB were $35.3 \%$ $(n=6), 41.2 \%(n=7)$ and $64.7 \%(n=11)$, respectively. Type I was observed in $58.3 \%$ and $52.9 \%$ in BiOSS and rDES groups, respectively, whereas the other types were less frequent (type II: $20.8 \%$ vs. $29.4 \%$; type III: $8.3 \%$ vs. $11.8 \%$; type IV: $12.5 \%$ vs. $5.9 \%$ ). In the BiOSS group most commonly restenosis type IA (focal, in MV) was observed $(28.6 \%)$, whereas in rDES restenosis type IC (focal, in SB) was most common, with an incidence of $17.7 \%$ (Figure 1).

In the BiOSS group 2 (8.3\%) restenosis cases were treated with CABG, 4 (16.7\%) with plain old ballon angioplasty/drug-eluting ballon (POBA/DEB) and 18 (75\%) with another DES implantation. In the rDES group 1 (5.9\%) restenosis case was treated with CABG, 4 (23.5\%) with POBA/ DEB and 12 (70.6\%) with another DES implantation.

At 12 months after the first ISR the death rates were $0,0,25 \%(n=1)$ and 0 for types I, II, III, IV, respectively; the $\mathrm{MI}$ rates were $4.3 \%(n=1), 0,0$ and 0 , whereas the TLR rates were $17.4 \%(n=4), 20 \%(n=2), 25 \%(n=1)$ and $50 \%(n=2)$. There were no statistical differences between BiOSS and rDES.

At 36 months after the first ISR the death rates were 4.3\% $(n=1), 0,25 \%(n=1)$ and 0 for types I, II, III, IV, respectively; the $\mathrm{MI}$ rates were $8.6 \%(n=2), 10 \%(n=1)$, 0 and 0 , whereas the TLR rates were $26.1 \%(n=6), 30 \%$ $(n=3), 25 \%(n=1)$ and $50 \%(n=2)$. There were no statistical differences between BiOSS and rDES.

\section{Discussion}

In-stent restenosis manifests in different angiographic patterns. We have proposed a classification which takes into account not only lesion length but also the location of the neointimal proliferation relative to the initially implanted stent in the bifurcation complex as well as to the stages of the $\mathrm{PCl}$ reflecting the proper stent apposition. There were no significant differences between BiOSS stents and rDES restenosis profile.

In the original Mehran classification 12-month clinical event rates were evenly high, without significant differences between groups regarding death or MI. However, a significant increase in TLR with increasing levels of ISR classification (class I, 19\%; class II, 35\%; class III, 50\%; and class IV, 83\%; $p<0.0001)$ was observed. This was caused by significantly increasing rates of $\mathrm{PCl}(15 \%, 26 \%$, $36 \%$, and $67 \%$ in classes I to IV, respectively; $p<0.0001$ ) as well as CABG $(4 \%, 8 \%, 14 \%$, and $17 \%$ in classes I to IV, respectively; $p<0.0001$ ) [3]. In our paper we obtained lower TLR rates. Although we treated bifurcation lesions characterized by higher failure rates, we used drug-eluting stents (mainly second generation), which perform better than bare metal stents available in 1999. Moreover, the procedure technique is quite different with FKB performed quite often and mandatory POT. Also, opposite to Mehran's initial paper we did not observe a very high rate of subsequent revascularizations after interventional therapy (ISR treatment) with currently available treatment modalities in patients presenting with higher ISR classes. Similar results were obtained both in the RIBS and RIBS II trials [11, 12].

In further studies, it would be of interest to verify whether the performance of the $\mathrm{PCl}$ with bifurcation lesions according to the European Bifurcation Club, especially performing POT or not, has an influence on the restenosis profile and the nature of such change [13].

\section{Conclusions}

In-stent restenosis presents with different angiographic patterns that might provide helpful prognostic information. There were no significant differences between the BiOSS stent and rDES restenosis profile in short- or long-term follow-up.

\section{Conflict of interest}

Robert J. Gil is a Balton consultant. Other authors declare no conflict of interest.

\section{References}

1. Alraies MC, Darmoch F, Tummala R, et al. Diagnosis and management challenges of in-stent restenosis in coronary arteries. World J Cardiol 2017; 9: 640-51.

2. Rzeszutko L, Siudak Z, Tokarek T, et al. Twelve months clinical outcome after bioresorbable vascular scaffold implantation in patients with stable angina and acute coronary syndrome. Data from the Polish National Registry. Adv Interv Cardiol 2016; 12: 108-15.

3. Mehran R, Dangas G, Abizaid AS, et al. Angiographic patterns of in-stent restenosis: classification and implications for long-term outcome. Circulation 1999; 100: 1872-8.

4. Gil RJ, Bil J, Kern A, et al. Comparison of dedicated BIOSS bifurcation stents with regular drug-eluting stents for coronary artery bifurcated lesions: pooled analysis from two randomized studies. Cardiol J 2018; 25: 308-16.

5. Gil RJ, Bil J, Dzavik V, et al. Regular drug-eluting stent vs. dedicated coronary bifurcation BiOSS expert stent: multicenter open-label randomized controlled POLBOS I trial. Can J Cardiol 2015; 31: 671-8.

6. Gil RJ, Bil J, Grundeken MJ, et al. Regular drug-eluting stents versus the dedicated coronary bifurcation sirolimus-eluting BiOSS LIM(R) stent: the randomised, multicentre, open-label, controlled POLBOS II trial. Eurolntervention 2016; 12: e1404-12. 
7. Bil J, Gil RJ, Vassilev D, et al. Dedicated bifurcation paclitaxel-eluting stent BiOSS Expert( $R)$ in the treatment of distal left main stem stenosis. J Interv Cardiol 2014; 27: 242-51.

8. Bil J, Gil RJ, Pawlowski T, et al. Assessment of vascular response to BiOSS LIM C((R)) stents vs Orsiro((R)) stents in the porcine coronary artery model. Cardiovasc Ther 2017; 35. doi: 10.1111/1755-5922.12267.

9. Bil J, Grundeken MJ, Pawlowski T, et al. Self-positioning properties of dedicated bifurcation coronary stent BiOSS LIM(R) in the eye of 3D optical coherence tomography. Minerva Cardioangiol 2017; 65: 194-6.

10. Thygesen K, Alpert JS, Jaffe AS, et al. Third universal definition of myocardial infarction. Circulation 2012; 126: 2020-35.

11. Alfonso F, Auge JM, Zueco J, et al. Long-term results (three to five years) of the Restenosis Intrastent: balloon angioplasty versus elective Stenting (RIBS) randomized study. J Am Coll Cardiol 2005; 46: 756-60.

12. Alfonso F, Perez-Vizcayno MJ, Hernandez R, et al. Long-term clinical benefit of sirolimus-eluting stents in patients with in-stent restenosis results of the RIBS-II (Restenosis Intra-stent: Balloon angioplasty vs. elective sirolimus-eluting Stenting) study. J Am Coll Cardiol 2008; 52: 1621-7.

13. Lassen JF, Burzotta F, Banning AP, et al. Percutaneous coronary intervention for the left main stem and other bifurcation lesions: $12^{\text {th }}$ consensus document from the European Bifurcation Club. Eurolntervention 2018; 13: 1540-53. 